\title{
Complexation of DNA with cationic surfactant
}

\author{
Paulo S. Kuhn, Marcia C. Barbosa, Yan Levin* \\ Instituto de Física, Universidade Federal do Rio Grande do Sul Caixa Postal 15051, CEP 91501-970, \\ Porto Alegre, RS, Brazil
}

Received 30 April 1999

\begin{abstract}
Transfection of an anionic polynucleotide through a negatively charged membrane is an important problem in genetic engineering. The direct association of cationic surfactant to DNA decreases the effective negative charge of the nucleic acid, allowing the DNA-surfactant complex to approach a negatively charged membrane. The paper develops a theory for solutions composed of polyelectrolyte, salt, and ionic surfactant. The theoretical predictions are compared with the experimental measurements. (c) 1999 Elsevier Science B.V. All rights reserved.
\end{abstract}

PACS: $05.70 . \mathrm{Ce} ; 61.20 . \mathrm{Qg} ; 61.25 . \mathrm{Hq}$

Keywords: Thermodynamic functions; Equations of state; Structure of associated liquids; Electrolytes; Molten salts; Macromolecular and polymer solutions; Polymer melts; Swelling

Gene therapy has increasingly captured public attention after the first gene transfer study in humans was completed in 1995 [1-3]. The procedure delivers a functional polynucleotide sequence into the cells of an organism affected by a genetic disorder. The gene delivery system that has been adopted in over $90 \%$ of the clinical trials to date is in the form of genetically engineered non-replicating retroviral or adenoviral vectors [2]. Unfortunately, the adverse response of the immune system has hindered application of virus-based gene therapy. New strategies are now being explored [4-19]. One of the approaches, pioneered by Felgner and Ringold [5], relies on association between the anionic nucleic acid and cationic lipid liposomes. The process of association neutralizes the excess negative charge of a polynucleotide, allowing the DNA-lipid complex to approach a negatively charged phospholipid membrane.

\footnotetext{
* Corresponding author. Fax: +55-51-31-91-762.

E-mail address: levin@if.ufrgs.br (Y. Levin)
} 


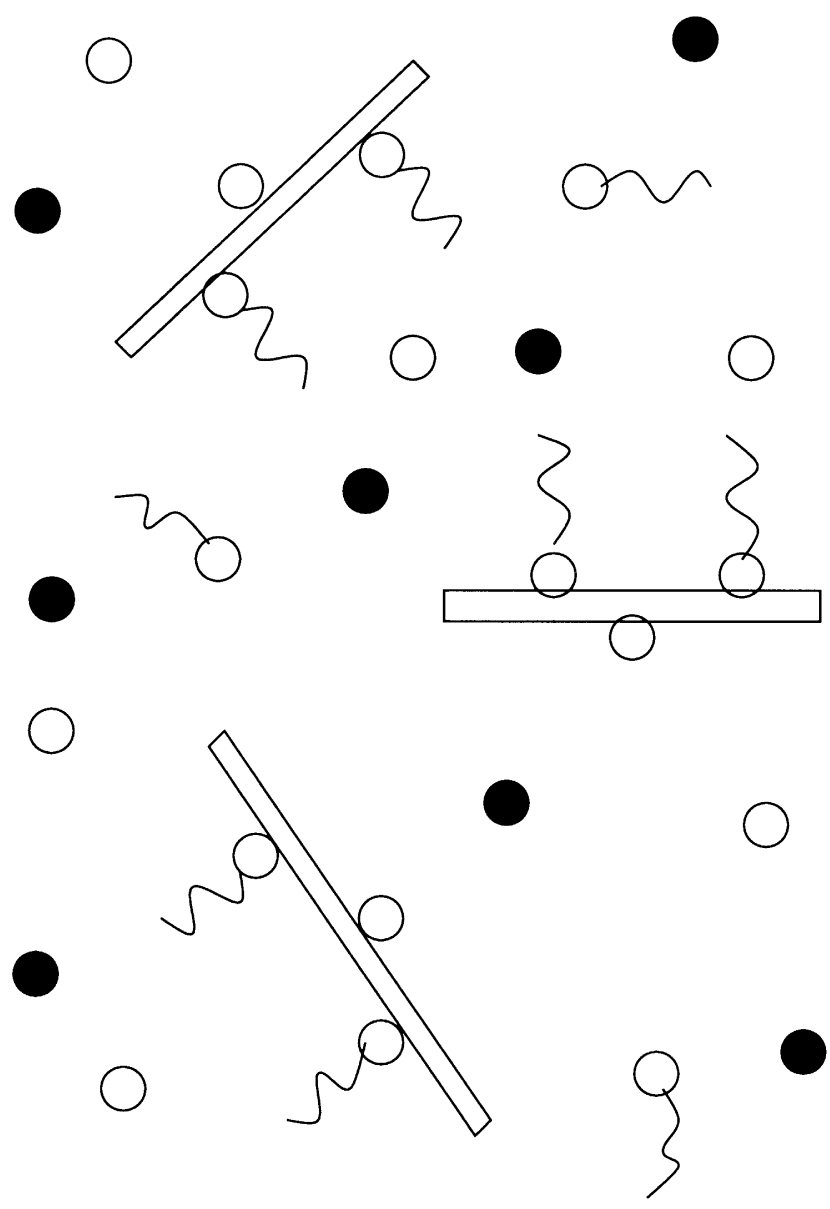

Fig. 1. Schematic Illustration of the Model: Complexes formed by DNA molecules (rectangles), counterions (solid circles) and flexible surfactant molecules (open circles with chains) are surrounded by free counterions, coions (open circles), and surfactant molecules.

Unfortunately, the cationic lipids and surfactants are toxic to an organism. A question that we will try to answer in this letter is: What is the minimum amount of cationic surfactant or lipid that is necessary to form a complex and how does this amount depend on various properties of a system?

We study a solution consisting of DNA segments of density $\rho_{\mathrm{DNA}}$, surfactants of density $\rho_{\text {surf }}$, and salt molecules of density $\rho_{\text {salt }}$ [21]. The solvent is idealized as a uniform medium of dielectric constant $D$. Since the DNA molecule has a large intrinsic rigidity, we model it as a cylinder of fixed length and diameter. When in solution, the $Z$ phosphate groups of the DNA strand become ionized, resulting in a net molecular charge, $-Z q$. An equivalent number of counterions of density $Z \rho_{\mathrm{DNA}}$ are released into solution preserving the overall charge neutrality. Similarly, the cationic surfactant 


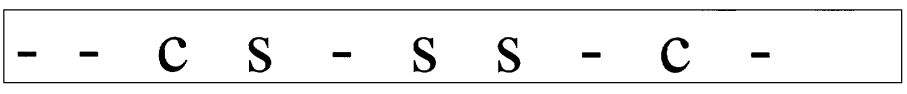

Fig. 2. Lattice model for forming a cluster. Each monomer can be either occupied by a counterion $(C)$, a surfactant $(S)$, or neither $(-)$. Interaction between the counterions is purely electrostatic, while two neighboring surfactant molecules gain energy due to hydrophobic effects.

molecule in an aqueous solution becomes ionized, producing a free negative ion and a flexible chain consisting of one positively charged hydrophilic head group and a neutral hydrophobic tail. The ions of salt, the counterions, and the negative ions dissociated from the surfactant are modeled as hard spheres with point charge located at the center. For simplicity, we shall call the negative ions, "coions", and the positive ions, "counterions" - independent of the species from which they are derived (see Fig. 1).

The strong electrostatic attraction between the counterions, cationic surfactant, and the DNA favors formation of clusters consisting of one DNA molecule and $n_{\text {count }}$ associated counterions, and $n_{\text {surf }}$ associated surfactants. The process of association neutralizes $n_{\text {surf }}+n_{\text {count }}$ phosphate groups of a DNA molecule, decreasing the net charge of a complex to, $q_{\text {complex }}=-\left(Z-n_{\text {surf }}-n_{\text {count }}\right) q$ (Fig. 1). Our task is to determine the values of $n_{\text {count }}$ and $n_{\text {surf }}$ which are thermodynamically favored, i.e. which minimize the overall Helmholtz free energy of solution.

For a dilute suspension, the main contributions to the free energy can be subdivided into three parts: the energy that it takes to construct an isolated complex, $F_{\text {association}}$; an energy that it takes to solvate this complex in the ionic sea, $F_{\text {solvation}}$; and the entropic energy of mixing, $F_{\text {mixing }}$.

To calculate the free energy of an isolated cluster, we use the following simplified model of a complex. Each monomer of a polyion is treated as free or occupied by a counterion or a surfactant (Fig. 2). We associate with each monomer $i$ two occupation variables $\sigma(i)$ and $\tau(i)$, such that $\sigma(t)=1$ if the site is occupied by a condensed counterion, and $\sigma(i)=0$ otherwise. The occupation number for surfactants, $\tau(i)$, behaves in a similar way. The free energy can now be calculated as a logarithm of the Boltzmann sum over all possible configurations of condensed counterions and surfactants along the polyion,

$$
\beta F_{\text {association }}=-\ln \sum_{v} \mathrm{e}^{-\beta E_{v}} .
$$

The energy of a given configuration $v$ can be subdivided into two parts, $E_{v}=E_{1}+E_{2}$, where the electrostatic contribution is

$$
E_{1}=\frac{q^{2}}{2} \sum_{i \neq j} \frac{[-1+\sigma(i)+\tau(i)][-1+\sigma(j)+\tau(j)]}{D|r(i)-r(j)|} .
$$

The energy $E_{2}$ arises from hydrophobicity of surfactant molecules. Clearly, when two adjacent sites are occupied by surfactants, the net exposure of hydrocarbon tails to water is reduced. We capture this effect by introducing an additional contribution to 
the overall energy of interaction, $E_{2}$, given by

$$
E_{2}=-\frac{\chi}{2} \sum_{\langle i \neq j\rangle} \tau(i) \tau(j),
$$

where the sum runs over the nearest neighbors. The parameter $\chi$, related to the decrease in overall energy due to the agglomeration of surfactants, is obtained from an independent experimental measurement of the energy that is required to move one surfactant molecule from a monolayer to the bulk [20].

The exact solution of even this one-dimensional "sub-problem" is rather difficult due to the long-ranged character of the Coulomb force. To proceed we could use a mean-field approximation, but while the mean-field theory works very well for long-range potentials, for one-dimensional systems with short-range forces, it can lead to unphysical instabilities [21]. In order to avoid this difficulty, we treat the long-range electrostatic part of the association free energy using a mean-field approximation, while performing an exact calculation for the short-range hydrophobic interaction.

Once a cluster, constructed in isolation, is introduced into solution, it gains an additional energy due to electrostatic interactions with the other entities. The free energy gained in the process of solvation can be obtained using the Debye-Hückel [21,22] theory. Let us fix the position of one cluster and ask what is the electrostatic potential $\Phi$ that this cluster feels as a result of the presence of all the other clusters, surfactants, counterions, and coions. To answer this question, consider the Poisson equation,

$$
\nabla^{2} \Phi=-\frac{4 \pi}{D} \rho_{q}
$$

To make the problem well posed, this equation must be provided with a closure which would relate the electrostatic potential, $\Phi$, to the net charge density, $\rho_{q}$. A simple closure motivated by ideas derived from the Debye-Hückel theory is to suppose that the free (unassociated) surfactants, counterions, and coions are distributed around the complex in accordance with the Boltzmann distribution, with other clusters providing a neutralizing background,

$$
\rho_{q}=q_{\text {complex }} \rho_{\text {DNA }}+q \rho_{\text {count }} \mathrm{e}^{-\beta q \Phi}-q \rho_{\text {coion }} \mathrm{e}^{+\beta q \Phi}+q \rho_{\text {surf }} \mathrm{e}^{-\beta q \Phi},
$$

where $\beta=1 /\left(k_{B} T\right)$.

Inserting (5) into (4) we obtain the Poisson-Boltzmann equation, which after linerization reduces to the familiar Helmholtz form. The linearization is justified since all the non-linearities are effectively included in the renormalization of DNA charge by the formation of clusters [23-25]. The Helmholtz equation can be solved analytically, yielding the electrostatic potential of a complex. The electrostatic free energy of solvation is obtained through the usual Debye charging process [22,21].

The free energy due to mixing of various species is a sum of individual entropic contributions,

$$
F_{\text {mixing }}=F_{\text {counterion }}+F_{\text {coion }}+F_{\text {surfactant }}+F_{\text {complex }} \text {. }
$$




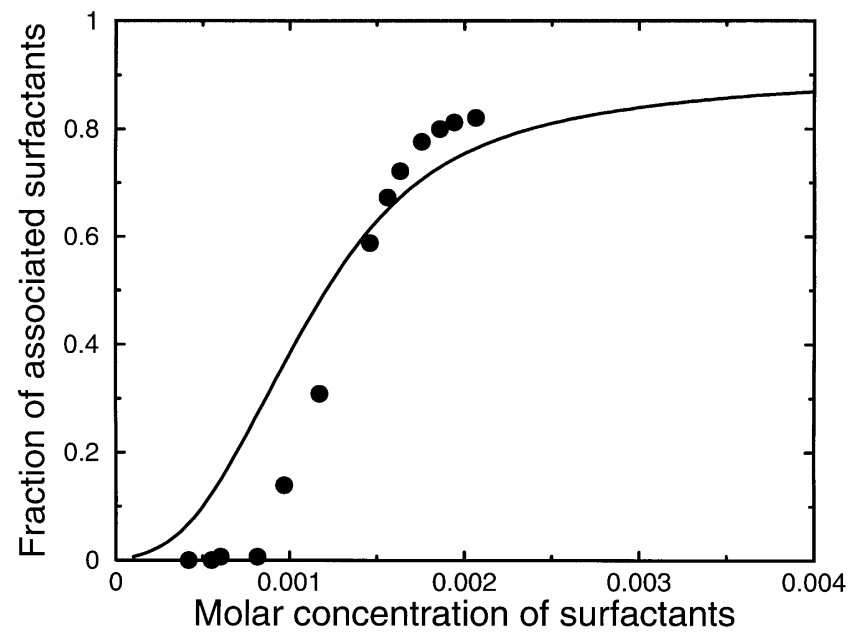

Fig. 3. Fraction of monomers along the DNA segment associated with surfactant molecules, $n_{\text {surf }} / Z$, as a function of the bulk density of surfactant, $\rho_{s}$. The solid line is derived from the solution of Eq. (8), while the circles are the experimental data [29]. The experimental value of the hydrophobicity parameter is $\chi=-3.5 k_{B} T$ [20]. The concentrations of DNA and of the added salt are $2 \times 10^{-6} \mathrm{M}$ and $18 \mathrm{mM}$, respectively. The length of a DNA segment is 220 base pairs.

The structure of each one of these terms is similar to that of an ideal gas and can be calculated using the Flory's theory of polymer melts [26]. For example, in the case of coions, the reduced free-energy density is $\beta F_{\text {coion }} / V=\rho_{\text {coion }} \ln \left(\phi_{\text {coion }} / \zeta\right)-\rho_{\text {coion }}$, where $\phi_{\text {coion }}$ is the volume fraction occupied by the negative microions, while $\zeta$ is a factor that takes into account the internal structure of each specie. For structureless particles such as coions and counterions, $\zeta=1$. For a flexible linear surfactant chain, $\zeta$ is the number of monomers comprising a molecule [26]. For a complex made of a DNA segment and condensed surfactants and counterions, $\zeta$ is related to the number of different configurations which can arise when $n_{\text {count }}$ counterions and $n_{\text {surf }}$ surfactant molecules associate to a DNA molecule. Minimizing the total free energy,

$$
F=F_{\text {mixing }}+F_{\text {association }}+F_{\text {solvation }},
$$

with respect to the number of associated counterions and surfactants,

$$
\frac{\partial F}{\partial n_{\text {cout }}}=\frac{\partial F}{\partial n_{\text {surf }}}=0,
$$

we find the thermodynamically preferred values for the number of condensed particles. We define a "surfoplex" to be a complex in which almost all of the DNA's phosphate groups are neutralized by the associated surfactant molecules. As mentioned earlier, we are interested in the minimum amount of cationic surfactant needed to transform naked DNA into surfoplexes. To this effect, we study the dependence of the number of condensed surfactant molecules, $n_{\text {surf }}$, on the bulk concentration of surfactant. Fig. 3 demonstrates the location of the cooperative binding transition associated with 
the formation of surfoplexes. The transition is a result of competition between the entropic, the electrostatic, and the hydrophobic interactions. Clearly, for small concentrations of surfactant, binding to the polyions is not thermodynamically favored, since the system can lower its free energy by keeping the surfactant in the bulk, thus gaining entropy. As the density of surfactant increases, the gain in electrostatic energy, due to binding, outweighs the loss of entropy due to confinement which, in any case, is largely compensated by the release of bound counterions [27,28]. The cooperativty predicted by our theory and observed in experiments [29,30] is due to the fact that once the first surfactant is bound, the binding of additional surfactants is strongly favored by the decrease in hydrophobic energy of the exposed hydrocarbon tails. The high degree of cooperativty allows us to clearly define how much surfactant is necessary to form a surfoplex. To check the predictions of our theory, we compare it to the recent experimental measurements [29] of binding isotherm in a solution of DNA, dodecyltrimethylammonium bromide, and salt, (Fig. 3). The agreement is encouraging specially since the theory does not have any fitting parameters.

We would like to acknowledge helpful conversations with Profs. K.A. Dawson, Michael E. Fisher, and Prof. H. E. Stanley. This work was supported in part by CNPq - Conselho Nacional de Desenvolvimento Científico e Tecnológico and FINEP Financiadora de Estudos e Projetos, Brazil.

\section{References}

[1] R.M. Blaese et al., Science 270 (1995) 475-480.

[2] M.J. Hope, B. Mui, S. Ansell, Q.F. Ahkong, Mol. Memb. Biol. 15 (1998) 1-14.

[3] W.F. Anderson, Nature 392 (1998) 25-30.

[4] P.L. Felgner, T.R. Gadek, M. Holm, M. Roman, M. Wentz, J.P. Northrop, M. Ringold, H. Danielsen, Proc. Natl. Acad. Sci. USA 84 (1987) 7413-7417.

[5] P.L. Felgner, G.M. Ringold, Nature 337 (1989) 387-388.

[6] T. Friedmann, Sci. Am. 276 (1997) 80-85.

[7] P.L. Felgner, Sci. Am. 276 (1997) 86-90.

[8] R. Langer, Science 249 (1990) 1527-1533.

[9] I.M. Verma, N. Somia, Nature 389 (1997) 239-242.

[10] S.T. Crooke, C.F. Bennett, Ann. Rev. Pharmacol. Toxicol. 36 (1996) 107-129.

[11] C.P. Hodgson, Biotechnology 13 (1995) 222-225.

[12] S. Akhtar, S. Agrawal, Trends Pharmacolog. Sci. 18 (1997) 12-18.

[13] C.F. Bennett, D. Kornbrust, S. Henry, K. Stecker, R. Howard, S. Cooper, S. Dutson, W. Hall, W.H.I. Jacoby, J. Pharmacol. Exp. Therapeut. 280 (1997) 988-1000.

[14] S.M. Sullivan, J. Invest. Dermatol. 103 (1994) 85S-89S.

[15] R.L. Juliano, S. Akhtar, Antisense Res. Dev. 2 (1992) 165-176.

[16] X. Gao, L. Huang, Gene Therapy 2 (1995) 710-722.

[17] J.G. Lewis, K.Y. Lin, A. Kothavale, W./M. Flanagan, M.D. Matteucci, R.B. DePrince, R.A. Mook, R.W. Hendren, R.W. Wagner, Proc. Natl. Acad. Sci. USA 93 (1996) 3176-3181.

[18] H. Farhood, R. Bottega, R.M. Epand, L. Huang, Biochem. Biophys. Acta 1111 (1992) 239-246.

[19] D.D. Lasic, H. Strey, M.C.A. Stuart, R. Podgornik, P.M. Frederik, J. Am. Chem. Soc. 119 (1997) $832-833$.

[20] J.N. Israelachvili, D.J. Mitchell, B.W. Ninham, J. Chem. Soc. Faraday Trans. 72 (1976) 1525-1568.

[21] P.S. Kuhn, Y. Levin, M.C. Barbosa, Chem. Phys. Lett. 298 (1998) 51-56.

[22] P.W. Debye, E. Hückel, Phys. Z. 24 (1923) 185-206. 
[23] Y. Levin, Europhys. Lett. 34 (1996) 405-410.

[24] S. Alexander et al., J. Chem. Phys. 80 (1984) 5776-5781.

[25] M.E. Fisher, Y. Levin, Phys. Rev. Lett. 71 (1993) 3826-3829.

[26] P. Flory, Principles of Polymer Chemistry, Cornell University Press, Ithaca, New York, 1971.

[27] G.S. Manning, J. Chem. Phys. 51 (1969) 924-933.

[28] G.S. Manning, Q. Rev. Biophys. 11 (1978) 179-246.

[29] A.V. Gorelov, E.D. Kudryashov, J.-C. Jacquier, D. McLoughlin, K.E. Dawson, Physica A 249 (1998) 216-225.

[30] K. Shirahama, K. Takashima, N. Takisawa, Bull. Chem. Soc. Jpn. 60 (1987) 43-47. 